\title{
El papel de la autoestima y la soledad en el uso problemático del smartphone: diferencias de género
}

\author{
Sergio Hidalgo-Fuentes ${ }^{1}$
}

\begin{abstract}
RESUMEN
El presente estudio investigó las relaciones entre la autoestima, la soledad y el uso problemático del smartphone y las diferencias de género. La muestra fue no probabilística y estuvo compuesta por 202 participantes (106 hombres y 96 mujeres), con edades comprendidas entre los 18 y los 58 años, que completaron las siguientes pruebas: Smartphone Addiction Scale, Social and Emotional Loneliness Scale for Adults y Escala de Autoestima de Rosenberg. Se calcularon los estadísticos descriptivos, la prueba U de MannWhitney, análisis de correlación bivariados y dos modelos de regresión múltiple jerárquica. Se hallaron diferencias significativas entre hombres y mujeres en el uso problemático del smartphone. Además, también se encontraron diferencias en función del género en las relaciones entre las variables. Los análisis de regresión mostraron que, entre las mujeres, la autoestima y la soledad social son predictores negativos del uso problemático del smartphone, mientras que la soledad romántica es un predictor positivo. El presente estudio contribuye a un mejor conocimiento de las causas del uso excesivo del smartphone.
\end{abstract}

Palabras Clave: autoestima, soledad, uso problemático del smartphone, diferencias de género.

\section{The role of self-esteem and loneliness in problematic smartphone use: gender differences}

\begin{abstract}
This study examined the relationships among self-esteem, loneliness and problematic smartphone use. The sample was non-probabilistic and consisted of 202 participants (106 male and 96 female), ranging in age from 18 to 58 years, who completed the following tests: Smartphone Addiction Scale, Social and Emotional Loneliness Scale for Adults and Rosenberg Self-Esteem Scale. Descriptive statistics, the Mann-Whitney U test, bivariate correlation analysis and two hierarchical multiple regression models were calculated. The results showed difference among male and female about problematic smartphone use. In addition, differences based on gender were also found in the relationships between the variables. Regression analysis showed that, among women, self-esteem and social loneliness are negative predictors of problematic smartphone use, while romantic loneliness is a positive predictor. The present study contributes to a better understanding of the causes of problematic smartphone use.
\end{abstract}

Keywords: self-esteem, loneliness, problematic smartphone use, gender differences.

\footnotetext{
1 Universidad de Valencia, España; sergio.hidalgo@uv.es; https://orcid.org/0000-0003-0842-4986
} 
Desde el lanzamiento del iPhone en 2007, las ventas de smartphones no han parado de incrementarse hasta alcanzar un total de tres mil seiscientos millones de usuarios a nivel mundial en 2020 (O’Dea, 2021). El uso del smartphone también goza de gran popularidad en España, donde el $78.9 \%$ de la población mayor de 15 años dispone de al menos uno de estos dispositivos (ONTSI, 2019).

Pese a las consecuencias negativas ligadas a un uso excesivo del smartphone, se ha generado un debate sobre el uso del término "adicción" aplicado a su utilización excesiva. Diversas investigaciones han encontrado un solapamiento entre la supuesta adicción al smartphone y el uso problemático de internet, redes sociales y aplicaciones de mensajería instantánea (Montag, Wegmann, Sariyska, Demetrovics, \& Brand, 2019; Sha, Sariyska, Riedl, Lachmann, \& Montag, 2019). Así, se consideraría el smartphone como un medio para el acceso a estos últimos y no un problema en sí mismo. Además, algunos autores afirman que las consecuencias negativas provocadas por la utilización excesiva del smartphone no son comparables a las causadas por otro tipo de adicciones, por lo que aconsejan la utilización de términos alternativos como "uso problemático" en lugar de hablar de adicción (Panova \& Carbonell, 2018). Por lo tanto, aunque en la literatura científica se hace mención indistintamente y como sinónimos a términos como "uso problemático del smartphone", "adicción al smartphone" o "uso excesivo del smartphone" (Thomée, 2018), en el presente trabajo se hará referencia a "uso problemático del smartphone" ya que este problema no se encuentra recogido como una adicción ni en el DSM-5 (American Psychiatric Association, 2013) ni en el CIE-11 (World Health Organization, 2018). El uso problemático del smartphone se ha convertido en un fenómeno global con una prevalencia del $23.67 \%$ entre estudiantes universitarios españoles (Ballestar-Tarín et al., 2020); del 62.6\% entre adolescentes filipinos (Buctot, Kim, \& Kim, 2020), del 29.8\% entre universitarios chinos (Chen et al., 2017), del 30.9\% entre adolescentes surcoreanos (Cha \& Seo, 2018), y del 44.7\% entre estudiantes universitarios indios (Kumar, Chandrasekaran, \& Brahadeeswari, 2019). En relación con el género, numerosos estudios han encontrado un mayor riesgo de uso problemático del smartphone entre mujeres (Amador-Licona, Carpio-Mendoza, Guízar-Mendoza, \& Rodríguez-Sánchez, 2019; Lee, Kim, \& Choi, 2017; Lopez-Fernandez, LosadaLopez, \& Honrubia-Serrano, 2015; Nayak, 2018; Randler et al., 2016). Algunos autores explican esta prevalencia en mujeres dado que ellas poseen una mayor orientación hacia las relaciones sociales (Lee et al., 2017; Weiser, 2000); así, las mujeres harian un uso principalmente social del smartphone, fundamentalmente a través de aplicaciones relacionadas con la mensajería instantánea y las redes sociales (Carbonell, Chamarro, Oberst, Rodrigo, \& Prades, 2018; Chen et al., 2017); mientras que entre los usuarios varones se encuentran patrones de uso más diversos (De-Sola, Rodríguez, \& Rubio, 2016). La literatura científica ha asociado el uso problemático del smartphone con numerosas consecuencias negativas, como la disminución del rendimiento académico (Amez \& Baert, 2020), la procrastinación académica (Yang, Asbury, $\&$ Griffiths, 2019), los altos niveles de estrés (Oraison, Nash-Dolby, Wilson, \& Malhotra, 2020), ansiedad (Hawi \& Samaha, 2017), la depresión (Alhassan et al., 2018), el bajo rendimiento laboral (Duke \& Montag, 2017) o la menor satisfacción con la vida (Lachmann et al., 2018).

En cuanto a la autoestima, esta ha sido definida como la "evaluación subjetiva del individuo sobre su valor como persona" (Trzesniewski, Donnellan, 
$\&$ Robins, 2013, p. 60). Diversos estudios han encontrado una relación negativa entre la autoestima y el uso problemático del smartphone (Işiklar, Şar, \& Durmuşcelebi, 2013; Kim \& Koh, 2018; Lee et al., 2018; Romero-Rodríguez, Aznar-Díaz, Marin-Marín, Soler-Costa, \& Rodríguez-Jiménez, 2020; Wang et al., 2017). Sin embargo, más investigaciones sobre el tema son necesarias ya que, por una parte, un estudio meta-analítico informó que esta asociación entre el uso problemático del smartphone y la autoestima es inconsistente (Elhai, Dvorak, Levine, \& Hall, 2017) y, por otra, no están claros los mecanismos que subyacen a esta relación.

En relación con la soledad, definida como un sentimiento de insatisfacción producido por la discrepancia cuando la cantidad o la calidad de las relaciones actuales de una persona no se corresponden a las que le gustaría mantener (Peplau \& Perlman, 1982; Peplau, Russell, \& Heim, 1979), es un fenómeno común en la sociedad contemporánea (Margalit, 2010). Estudios anteriores han vinculado altos niveles de soledad y uso problemático del smartphone (Bian \& Leung, 2015; Darcin et al., 2016; Mahapatra, 2019; Wang, Sigerson, Jiang, \& Cheng, 2018). Esta relación podría estar provocada por la asociación entre la soledad y bajas habilidades sociales (Lodder, Goossens, Scholte, Engels, \& Verhagen, 2016; Segrin, 2019; Wong, Yeung, \& Lee, 2018), lo que provocaria que las personas solitarias se sintieran más cómodas relacionándose con otros mediante un dispositivo digital que cara a cara.

El objetivo del presente estudio fue investigar la relación entre el uso problemático del smartphone, la autoestima y la soledad, así como también examinar si estas relaciones dependen del género del usuario. Por tanto, los objetivos específicos de este trabajo fueron: (1) evaluar las diferencias de género en el uso problemático del smartphone, la autoestima y la soledad; (2) analizar las relaciones entre el uso problemático del smartphone, la autoestima y la soledad en función del género; (3) evaluar la capacidad predictiva de la autoestima y la soledad sobre el uso problemático del smartphone en hombres y mujeres.

\section{MÉTODO}

\section{Participantes}

La muestra, recogida mediante un muestreo incidental no aleatorio, estuvo formada por 202 participantes, compuesta por 106 hombres (52.5\%) y 96 mujeres (47.5\%), con edades comprendidas entre los 18 y 58 años $(M=24.73$; $D T=6.90$ ). Los participantes debian ser mayores de edad y ser usuarios de smartphone.

\section{Materiales}

El uso problemático del smartphone fue cuantificado a través de la prueba Smartphone Addiction Scale-Short Version (SAS-SV; Kwon, Kim, Cho, \& Yang, 2013). La escala, diseñada para identificar el nivel de riesgo de adicción a los smartphones, está formada por 10 ítems (e.g., "La gente de mi alrededor me dice que uso demasiado mi smartphone") con formato de respuesta tipo likert de seis puntos (desde 1 totalmente en desacuerdo a 6 totalmente de acuerdo). El rango de puntuación total de la escala se encuentra entre 10 y 60 (una mayor puntuación se asocia a una mayor prevalencia de adicción al smartphone). Para este estudio, se empleó la adaptación al español realizada por Lopez-Fernandez (2017). Se obtuvo en esta muestra una consistencia interna adecuada $(a=.91)$. 
La soledad emocional y social fueron evaluada mediante la versión corta de la prueba Social and Emotional Loneliness Scale for Adults (SESLA-S; DiTommaso, Brannen, \& Best, 2004). Para el presente trabajo, se utilizó la adaptación al español llevada a cabo por Yárnoz (2008) que evalúa la soledad a través de tres factores: soledad social, o grado en que los sujetos perciben escasas relaciones sociales o de amistad; soledad romántica, que refleja la falta de relaciones afectivas íntimas; y soledad familiar, que manifiesta una baja percepción de apoyo por parte del entorno familiar. La escala está formada por 15 items (e.g., "No tengo ningún amigo que me comprenda, pero me gustaría tenerlo"), cinco para cada uno de los factores, con formato de respuesta tipo likert de siete puntos (desde 1 fuertemente en desacuerdo a 7 fuertemente de acuerdo). Para cada uno de los factores, la puntuación oscila entre 5 y 35, asociándose las mayores puntuaciones a una mayor soledad autopercibida. La adaptación utilizada presentó una satisfactoria consistencia interna en los tres factores: soledad familiar $(a=.90)$, soledad romántica $(a=.92)$ y soledad social $(a=.91)$.

Para la evaluación de la autoestima, se utilizó la versión adaptada al español (Martín-Albo, Núñez, Navarro, \& Grijalvo, 2007) de la Escala de Autoestima de Rosenberg (Rosenberg, 1965). Esta prueba evalúa la autoestima global mediante los sentimientos positivos y negativos hacia uno mismo. La escala está compuesta de 10 ítems (e.g., "Tengo una actitud positiva hacia mí mismo") que se responden sobre una escala likert de cuatro puntos (desde 1 totalmente en desacuerdo a 4 totalmente de acuerdo). La puntuación global oscila en un rango de 10 a 40, y las puntuaciones más elevadas se asocian a una mayor autoestima. En este estudio, la adaptación utilizada presentó una buena consistencia interna $(a=.92)$.

Junto a las pruebas estandarizadas se recogió información sociodemográfica (sexo y edad) de los participantes.

\section{Diseño y procedimiento}

El diseño de investigación es de tipo predictivo transversal. Los participantes fueron reclutados mediante una doble estrategia. Por una parte, $\mathrm{y}$ tras recabar el permiso de sus administradores, se publicaron invitaciones a participar en el estudio en diversos foros de internet relacionados con las nuevas tecnologías. Por otro lado, también se informó a alumnos de una universidad pública española de la realización del estudio solicitando su participación. En ambos casos, las solicitudes de participación contenian información sobre el estudio así como sobre su carácter voluntario, anónimo y confidencial. Las solicitudes incluian un enlace al protocolo del estudio que contenía las pruebas realizado mediante la aplicación Google Forms. Antes de comenzar a contestar las pruebas, la primera página del protocolo presentaba, de nuevo, información sobre la investigación y los sujetos debian dar su consentimiento a participar marcando un item creado a tal efecto. No se ofrecieron recompensas por la participación en la investigación.

El análisis de los datos se realizó mediante el programa IBM SPSS Statistics versión 26. La fiabilidad de las pruebas utilizadas fue calculada mediante el a de Cronbach. Se analizó la normalidad de las variables a través de la prueba de Kolmogorov-Smirnov y se obtuvieron $p$-valores inferiores a .05 en todas ellas, lo que refleja la ausencia de normalidad. Además, se calcularon los estadísticos descriptivos y las correlaciones bivariadas rho de Spearman de las distintas variables y se estudiaron las diferencias en función del género con 
la prueba de la U de Mann-Whitney. Para valorar el tamaño del efecto de las diferencias entre hombres y mujeres, se utilizó la fórmula $r=Z / \sqrt{ } \mathrm{N}$ (Fritz, Morris, $\&$ Richler, 2012) que se interpreta según los siguientes rangos: .1, pequeño; .3, medio; y .5 grande. Para cuantificar la capacidad predictiva de la autoestima y la soledad sobre el uso problemático del smartphone, se realizaron dos análisis de regresión jerárquica: uno para los hombres y otro para las mujeres, entrando la autoestima en el primer paso del modelo y los tres factores de la escala de soledad en el segundo paso.

\section{RESULTADOS}

En la Tabla 1, se presentan los estadísticos descriptivos, así como las diferencias entre hombres y mujeres en las variables del estudio calculadas mediante la prueba de Mann-Whitney. Como se puede observar, existen diferencias estadísticamente significativas en función del género en el nivel de uso problemático del smartphone $(z=-6.80 ; p<.001)$ dado que las mujeres muestran un nivel mayor con un tamaño del efecto grande $(r=.48)$. Asimismo, también se hallaron diferencias significativas entre hombres y mujeres en las variables autoestima $(z=-2.11 ; p=.035)$, soledad romántica $(z=-2.15 ; p=.031)$ y soledad social $(z=-2.24 ; p=.025)$, en todos los casos con un tamaño del efecto pequeño y puntuaciones más altas entre los hombres de la muestra.

Tabla 1.

Estadísticos descriptivos y diferencias en función del género.

\begin{tabular}{lcccccccc}
\hline & \multicolumn{3}{c}{ Hombres } & \multicolumn{2}{c}{ Mujeres } & \multicolumn{4}{c}{ Diferencias } \\
\cline { 2 - 9 } & $\boldsymbol{M}$ & $\boldsymbol{D T}$ & $\boldsymbol{M}$ & $\boldsymbol{D T}$ & $\boldsymbol{U}$ & $\boldsymbol{Z}$ & $\boldsymbol{p}$ & $\begin{array}{c}\text { Tamaño } \\
\text { del efecto }\end{array}$ \\
\cline { 2 - 9 } $\begin{array}{l}\text { Uso problemático } \\
\text { del smartphone }\end{array}$ & 24.42 & 10.23 & 35.30 & 10.10 & 2270.5 & -6.80 & $<.001$ & -0.48 \\
Autoestima & 28.52 & 7.12 & 26.29 & 7.56 & 4215 & -2.11 & .035 & -0.15 \\
Soledad familiar & 13.11 & 7.19 & 11.66 & 6.71 & 4338 & -1.81 & .070 & -0.13 \\
Soledad romántica & 22.64 & 11.46 & 20.43 & 9.94 & 4196.5 & -2.15 & .031 & -0.15 \\
Soledad social & 13.95 & 7.54 & 11.98 & 7.22 & 4160 & -2.24 & .025 & -0.16 \\
\hline
\end{tabular}

Los análisis de correlación (véase Tabla 2) muestran como para el grupo de mujeres el uso problemático del smartphone presenta correlaciones estadísticamente significativas de signo negativo con la autoestima ( $r h o=-.21$; $p<.05$ ) y la soledad social ( $r h o=-.23 ; p<.05)$, y de signo positivo con la soledad romántica (rho $=.236 ; p<.05$ ). En el caso de los hombres, se hallaron correlaciones estadisticamente significativas entre el uso problemático del smartphone y la soledad romántica ( $r h o=.230 ; p<.05)$ y la soledad social (rho $=.301 ; p<.01)$.

Por último, con el objetivo de valorar la capacidad predictiva tanto de la autoestima como de la soledad sobre el uso problemático del smartphone en hombres y mujeres, se llevaron a cabo análisis de regresión jerárquica introduciendo, en el primer modelo, la autoestima y, en el segundo modelo, las tres dimensiones de soledad evaluadas (véase tabla 3). Se comprobaron los supuestos de linealidad, a través de los diagramas de dispersión parcial para cada variable independiente; normalidad de los residuos mediante la prueba de Kolmogorov-Smirnov; homocedasticidad por medio de la prueba de Levene; independencia de los residuos por medio de la prueba de Durbin-Watson y se 
descartó la colinealidad, comprobando los valores de inflación de varianza y los indices de tolerancia. Para los hombres, ni la autoestima ni la soledad resultaron predictores significativos del uso problemático del smartphone. En el caso de las mujeres, por un lado, la introducción de la autoestima en el primer modelo del análisis resultó significativa y explicó el $5.1 \%$ de la varianza del uso problemático del smartphone. Por otro lado, la introducción de la soledad también fue significativa, aumentando la varianza explicada en un $12.4 \%$ adicional. Así, la soledad romántica se presentó como un predictor significativo de signo positivo y la soledad social como un predictor significativo de signo negativo.

Tabla 2.

Correlaciones bivariadas de Spearman en función del género.

1. Uso problemático del smartphone

\begin{tabular}{ccccc}
1 & 2 & 3 & 4 & 5 \\
\hline- & $-.21^{*}$ & -.06 & $.24^{*}$ & $-.23^{*}$ \\
-.14 & - & $-.37^{* *}$ & $-.32^{* *}$ & -.17 \\
.14 & $-.37^{* *}$ & - & .08 & $.39^{* *}$ \\
$.23^{*}$ & $-.52^{* *}$ & $.31^{* *}$ & - & -.04 \\
$.30^{* *}$ & $-.31^{* *}$ & $.60^{* *}$ & $.27^{* *}$ & - \\
\hline
\end{tabular}

2. Autoestima

os de la muestra de hombres; encima de la

3. Soledad familiar

4. Soledad romántica

5. Soledad social

Nota. Debajo de la diagonal se presentan los resultados de la

* $p<.05 ;{ }^{* *} p<.01$

Tabla 3.

Regresión múltiple jerárquica: contribución de la autoestima (Paso 1) y la soledad (Paso 2) al uso problemático del smartphone en función del género.

\begin{tabular}{|c|c|c|c|c|c|c|c|}
\hline & \multicolumn{3}{|c|}{ Hombres } & & \multicolumn{3}{|c|}{ Mujeres } \\
\hline & $\beta$ & $R^{2}$ & $\Delta R^{2}$ & & $B$ & $R^{2}$ & $\Delta R^{2}$ \\
\hline $\begin{array}{l}\text { Modelo } 1 \\
\text { Autoestima }\end{array}$ & -.19 & .04 & & $\begin{array}{l}\text { Modelo } 1 \\
\text { Autoestima }\end{array}$ & $-.23^{*}$ & $.05^{\star}$ & \\
\hline $\begin{array}{l}\text { Modelo } 2 \\
\text { Autoestima } \\
\text { Soledad familiar } \\
\text { Soledad romántica } \\
\text { Soledad social }\end{array}$ & $\begin{array}{l}-.11 \\
-.07 \\
.07 \\
.23\end{array}$ & .08 & .04 & $\begin{array}{l}\text { Modelo } 2 \\
\text { Autoestima } \\
\text { Soledad familiar } \\
\text { Soledad romántica } \\
\text { Soledad social }\end{array}$ & $\begin{array}{l}-.24^{*} \\
-.12 \\
.21^{*} \\
-.22^{*}\end{array}$ & $.18^{* *}$ & $.12^{\star \star}$ \\
\hline
\end{tabular}

\section{COMENTARIOS}

En relación con el primer objetivo del estudio, los análisis realizados mediante la prueba U de Mann-Whitney muestran cómo las mujeres presentan, en comparación con los hombres, una puntuación significativamente superior y con un tamaño del efecto grande de uso problemático del smartphone. Este resultado es congruente con lo informado en investigaciones anteriores (Amador-Licona et al., 2019; Lee et al., 2017; Lopez-Fernandez et al., 2015; Nayak, 2018; Randler et al., 2016). La mayor probabilidad de hacer un uso excesivo del smartphone entre las mujeres podría ser provocada por el hecho de que las mujeres sientan mayor ansiedad en situaciones sociales que los hombres (Asher, Asnaani, \& Aderka, 2017), por lo que podrian utilizar el smartphone con más frecuencia para establecer y mantener contactos sociales con el objetivo de reducir dicha ansiedad (Van Deursen, Bolle, Hegner, \& 
Kommers, 2015). En relación con las diferencias de género en las variables independientes, se han encontrado diferencias estadísticamente significativas tanto en la autoestima como en la soledad romántica y familiar; en los tres casos con puntuaciones superiores, aunque con un tamaño del efecto pequeño, a favor de los hombres. La mayor autoestima encontrada en los hombres es un resultado esperable y coherente con la literatura cientifica. Un estudio realizado con participantes de 48 países halló que en todos ellos los hombres tenian una mayor autoestima que las mujeres (Bleidorn et al., 2016). Esta brecha de género a favor de los hombres se ha reportado en numerosos estudios con muestras muy diversas (Bachman, O'Malley, Freedman-Doan, Trzesniewski, \& Donnellan, 2011; Helwig \& Ruprecht, 2017; Manne-Goehler et al., 2020; McMullin \& Cairney, 2004; Puskar et al., 2010). Por su parte, la mayor soledad reportada por los varones coincide con los resultados de un reciente estudio realizado con participantes de 237 países que encontró una mayor soledad percibida por parte de los hombres (Barreto et al., 2021). Sin embargo, un metaanálisis realizado por Maes, Qualter, Vanhalst, Van den Noortgate y Goossens (2019) no encontró evidencias de que existieran diferencias en el nivel de soledad en función del género.

En cuanto al segundo objetivo, las principales diferencias encontradas en los análisis de correlación radican en que, en el caso de los hombres, la autoestima no mantiene relación estadísticamente significativa con el uso problemático del smartphone mientras que en las mujeres sí. Además, la soledad social correlaciona con el uso problemático del smartphone de manera positiva entre los hombres y de manera negativa entre las mujeres.

Por último, en relación con el tercer objetivo, los análisis de regresión muestran que, en el caso de los hombres ni la autoestima ni la soledad resultaron predictores significativos mientras que, entre las mujeres la autoestima y la soledad social predicen de manera negativa parte de la varianza del uso problemático del smartphone. En otras palabras, a menor autoestima y menor soledad social percibida, mayor uso del smartphone. La soledad romántica funciona como predictor positivo, por lo que la falta de relaciones intimas percibidas por las mujeres predice un uso excesivo del smartphone. Las mujeres utilizan sus dispositivos con una orientación básicamente social, mientras que los hombres hacen un uso más diverso que incluye la comunicación, los videojuegos, los juegos de azar, etc. (Chen et al., 2017; Lee, Chang, Lin, \& Cheng, 2014; Van Deursen et al., 2015). Esto es congruente con que la soledad no funcione como predictor en el caso de los hombres y que una baja soledad social, es decir, mantener una buena red de amistades, funcione como predictor de un mayor uso del smartphone entre las mujeres. El hecho de que la falta de relaciones intimas sentimentales sirva como predictor de la utilización problemática del smartphone podría deberse a que la falta de pareja aumente la frecuencia de comunicaciones con el grupo de amigos, ya que los compañeros sentimentales a menudo reemplazan a los amigos como principales figuras de apoyo (Larson, 1999). Que la baja autoestima prediga parte de la varianza del uso problemático del smartphone entre mujeres estaría en consonancia con lo expuesto por Billieux (2012), que encontró que aquellas personas con menor autoestima muestran preferencia por la comunicación indirecta.

A pesar de que esta investigación contribuye a aumentar el conocimiento de las variables asociadas el uso problemático del smartphone, no se encuentra exenta de limitaciones. Primeramente, al tratarse de un estudio transversal no 
se pueden establecer relaciones de causalidad entre las distintas variables ni evaluar la evolución de las relaciones encontradas a lo largo del tiempo, por lo que sería interesante continuar investigando en esta línea mediante estudios longitudinales. En segundo lugar, el muestreo es de tipo no probabilístico, lo que implica que hay que ser especialmente cauto a la hora de generalizar los resultados obtenidos. Por último, las pruebas utilizadas son de tipo autoinforme por lo que, a pesar de que este tipo de medidas han probado ser eficaces para evaluar el comportamiento, sería conveniente medir estas variables sobre una base conductual de cara a próximas investigaciones (Podsakoff, MacKenzie, Lee, \& Podsakoff, 2003), como así también mejorar la precisión de los datos relacionados con los patrones de uso del smartphone mediante herramientas de monitorización de frecuencia y duración del uso de aplicaciones. Pese a las limitaciones comentadas, el presente estudio contribuye a un mayor entendimiento del fenómeno del uso problemático del smartphone, que ha sido calificado por la Organización Mundial de la Salud como un problema de salud pública (World Health Organization, 2015); identificando, por un lado, la autoestima y la soledad social como factores predictores negativos de este fenómeno entre las mujeres $\mathrm{y}$, por el otro, la soledad romántica como un predictor positivo del uso excesivo del smartphone. Así, intervenciones centradas en aumentar la autoestima entre las mujeres podrian servir para reducir la prevalencia del uso problemático del smartphone, así como las consecuencias negativas asociadas.

\section{REFERENCIAS}

Alhassan, A. A., Alqadhib, E. M., Taha, N. W., Alahmari, R. A., Salam, M., \& Almutairi, A. F. (2018). The relationship between addiction to smartphone usage and depression among adults: a cross sectional study. BMC psychiatry, 18(1), 1-8. https://doi.org/10.1186/s12888-018-1745-4

Amador-Licona, N., Carpio-Mendoza, J. J., Guizar-Mendoza, J. M., \& Rodriguez-Sánchez, P. (2019). Autopercepción del Uso Problemático del Teléfono Móvil en Estudiantes Universitarios de Acuerdo a su Sexo. Cuadernos Hispanoamericanos de Psicologia, 19(2), 1-16. https://doi.org/10.18270/chps..v19i2.3134

American Psychiatric Association. (2013). Diagnostic and statistical manual of mental disorders: DSM-5. Washington, DC: American Psychiatric Publishing. https://doi.org/10.1176/appi.books.9780890425596

Amez, S. \& Baert, S. (2020). Smartphone use and academic performance: A literature review. International Journal of Educational Research, 103, 101618. https://doi.org/10.1016/j.ijer.2020.101618

Asher, M., Asnaani, A., \& Aderka, I. M. (2017). Gender differences in social anxiety disorder: A review. Clinical psychology review, 56, 1-12. https://doi.org/10.1016/j.cpr.2017.05.004

Bachman, J. G., O'Malley, P. M., Freedman-Doan, P., Trzesniewski, K. H., \& Donnellan, M. B. (2011). Adolescent self-esteem: Differences by race/ethnicity, gender, and age. Self and Identity, 10(4), 445-473. https://doi.org/10.1080/15298861003794538

Ballestar-Tarín, M. L., Simó-Sanz, C., Chover-Sierra, E., Saus-Ortega, C., Casal-Angulo, C., \& MartínezSabater, A. (2020). Self-Perception of dependence as an indicator of smartphone addictionEstablishment of a cutoff point in the SPAI-Spain Inventory. International Journal of Environmental Research and Public Health, 17(11), 3838. https://doi.org/10.3390/ijerph17113838

Barreto, M., Victor, C., Hammond, C., Eccles, A., Richins, M. T., \& Qualter, P. (2021). Loneliness around the world: Age, gender, and cultural differences in loneliness. Personality and Individual Differences, 169, 110066. https://doi.org/10.1016/j.paid.2020.110066

Bian, M. \& Leung, L. (2015). Linking loneliness, shyness, smartphone addiction symptoms, and patterns of smartphone use to social capital. Social science computer review, 33(1), 61-79. https://doi.org/10.1177/0894439314528779

Billieux, J. (2012). Problematic use of the mobile phone: a literature review and a pathways model. Current Psychiatry Reviews, 8(4), 299-307. https://doi.org/10.2174/157340012803520522

Bleidorn, W., Arslan, R. C., Denissen, J. J., Rentfrow, P. J., Gebauer, J. E., Potter, J., \& Gosling, S. D. (2016). Age and gender differences in self-esteem-A cross-cultural window. Journal of personality and social psychology, 111(3), 396. https://doi.org/10.1037/pspp0000078

Buctot, D. B., Kim, N., \& Kim, J. J. (2020). Factors associated with smartphone addiction prevalence and its predictive capacity for health-related quality of life among Filipino adolescents. Children and Youth Services Review, 110, 104758. https://doi.org/10.1016/j.childyouth.2020.104758

Carbonell, X., Chamarro, A., Oberst, U., Rodrigo, B., \& Prades, M. (2018). Problematic use of the Internet and smartphones in university students: 2006 - 2017. International Journal of Environmental Research and Public Health, 15(3), 1-13. https://doi.org/10.3390/ijerph15030475 
Cha, S. S. \& Seo, B. K. (2018). Smartphone use and smartphone addiction in middle school students in Korea: Prevalence, social networking service, and game use. Health psychology open, 5(1), 1-15. https://doi.org/10.1177/2055102918755046

Chen, B., Liu, F., Ding, S., Ying, X., Wang, L., \& Wen, Y. (2017). Gender differences in factors associated with smartphone addiction: a cross-sectional study among medical college students. BMC psychiatry, 17(1), 1-9. https://doi.org/10.1186/s12888-017-1503-z

Darcin, A. E., Kose, S., Noyan, C. O., Nurmedov, S., Y1lmaz, O., \& Dilbaz, N. (2016). Smartphone addiction and its relationship with social anxiety and loneliness. Behaviour \& Information Technology, 35(7), 520525. https://doi.org/10.1080/0144929X.2016.1158319

De-Sola, J., Rodríguez, F., \& Rubio, G. (2016). Cell-phone addiction: A review. Frontiers in psychiatry, 7, 175. https://doi.org/10.3389/fpsyt.2016.00175

DiTommaso, E., Brannen, C., \& Best, L. A. (2004). Measurement and validity characteristics of the short version of the social and emotional loneliness scale for adults. Educational and Psychological Measurement, 64, 99-119. https://doi.org/10.1177/0013164403258450

Duke, É. \& Montag, C. (2017). Smartphone addiction, daily interruptions and self-reported productivity. Addictive behaviors reports, 6, 90-95. https://doi.org/10.1016/j.abrep.2017.07.002

Elhai, J. D., Dvorak, R. D., Levine, J. C., \& Hall, B. J. (2017). Problematic smartphone use: A conceptual overview and systematic review of relations with anxiety and depression psychopathology. Journal of affective disorders, 207, 251-259. https://doi.org/10.1016/j.jad.2016.08.030

Fritz, C. O., Morris, P. E., \& Richler, J. J. (2012). Effect size estimates: current use, calculations, and interpretation. Journal of experimental psychology: General, 141(1), 2. https://doi.org/10.1037/a0024338

Hawi, N. S. \& Samaha, M. (2017). Relationships among smartphone addiction, anxiety, and family relations. $\begin{array}{lllll}\text { Behaviour \& Information } \quad \text { Technology, } & \text { 36(10), }\end{array}$ https://doi.org/10.1080/0144929X.2017.1336254

Helwig, N. E. \& Ruprecht, M. R. (2017). Age, gender, and self-esteem: A sociocultural look through a nonparametric lens. Archives of Scientific Psychology, 5(1), 19. https://doi.org/10.1037/arc0000032

Işiklar, A., Şar, A., \& Durmuşcelebi, M. (2013). An investigation of the relationship between high-school students' problematic mobile phone use and their self-esteem levels. Education, 134(1), 9-14.

Kim, E. \& Koh, E. (2018). Avoidant attachment and smartphone addiction in college students: The mediating effects of anxiety and self-esteem. Computers in Human Behavior, 84, 264-271. https://doi.org/10.1016/j.chb.2018.02.037

Kumar, V. A., Chandrasekaran, V., \& Brahadeeswari, H. (2019). Prevalence of smartphone addiction and its effects on sleep quality: A cross-sectional study among medical students. Industrial psychiatry journal, 28(1), 82. https://doi.org/10.4103/ipj.ipj_56_19

Kwon, M., Kim, D. J., Cho, H., \& Yang, S. (2013). The smartphone addiction scale: development and validation of a short version for adolescents. PloS one, 8(12), e83558. https://doi.org/10.1371/journal.pone.0083558

Lachmann, B., Sindermann, C., Sariyska, R. Y., Luo, R., Melchers, M. C., Becker, B., ... \& Montag, C. (2018). The role of empathy and life satisfaction in internet and smartphone use disorder. Frontiers in Psychology, 9, 398. https://doi.org/10.3389/fpsyg.2018.00398

Larson, R. (1999). The uses of loneliness in adolescence. In K. Rotenberg \& S. Hymel (Eds.), Loneliness in childhood and adolescence (pp. 296-322). New York: Cambridge University Press. https://doi.org/10.1017/CBO9780511551888.012

Lee, H., Kim, J. W., \& Choi, T. Y. (2017). Risk factors for smartphone addiction in Korean adolescents: smartphone use patterns. Journal of Korean medical science, 32(10), 1674. https://doi.org/10.3346/jkms.2017.32.10.1674

Lee, J., Sung, M. J., Song, S. H., Lee, Y. M., Lee, J. J., Cho, S. M., Park, M. K., \& Shin, Y. M. (2018). Psychological factors associated with smartphone addiction in south korean adolescents. The Journal of Early Adolescence, 38(3), 288-302. https://doi.org/10.1177/0272431616670751

Lee, Y. K., Chang, C. T., Lin, Y., \& Cheng, Z. H. (2014). The dark side of smartphone usage: Psychological traits, compulsive behavior and technostress. Computers in human behavior, 31, 373-383. https://doi.org/10.1016/j.chb.2013.10.047

Lodder, G. M. A., Goossens, L., Scholte, R. H. J., Engels, R. C. M. E., \& Verhagen, M. (2016). Adolescent loneliness and social skills: Agreement and discrepancies between self-, meta-, and peer-evaluations. Journal of youth and adolescence, 45(12), 2406-2416. https://doi.org/10.1007/s10964-016-0461-y

Lopez-Fernandez, O. (2017). Short version of the Smartphone Addiction Scale adapted to Spanish and French: Towards a cross-cultural research in problematic mobile phone use. Addictive behaviors, 64, 275-280. https://doi.org/10.1016/j.addbeh.2015.11.013

Lopez-Fernandez, O., Losada-Lopez, J. L., \& Honrubia-Serrano, M. L. (2015). Predictors of problematic Internet and mobile phone usage in adolescents. Aloma: revista de psicologia, ciències de l'educació $i$ de l'esport Blanquerna, 33(2), 49-58. https://doi.org/10.51698/aloma.2015.33.2.49-58

Maes, M., Qualter, P., Vanhalst, J., Van den Noortgate, W., Goossens, L., \& Kandler, C. (2019). Gender Differences in Loneliness across the Lifespan: A Meta-Analysis. European Journal of Personality, 33(6), 642-654. https://doi.org/10.1002/per.2220

Mahapatra, S. (2019). Smartphone addiction and associated consequences: Role of loneliness and self-

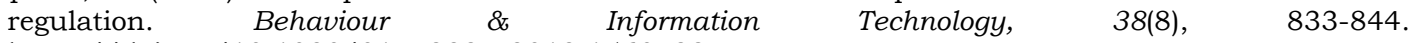
https://doi.org/10.1080/0144929X.2018.1560499

Manne-Goehler, J., Freund, K. M., Raj, A., Kaplan, S. E., Terrin, N., Breeze, J. L., \& Carr, P. L. (2020). Evaluating the role of self-esteem on differential career outcomes by gender in academic medicine. Academic Medicine, 95(10), 1558-1562. https://doi.org/10.1097/ACM.0000000000003138 
Margalit, M. (2010). Lonely children and adolescents: Self-perceptions, social exclusion, and hope. New York: Springer. https://doi.org/10.1007/978-1-4419-6284-3

Martín-Albo, J., Núñez, J. L., Navarro, J. G., \& Grijalvo, F. (2007). The Rosenberg Self-Esteem Scale: translation and validation in university students. The Spanish journal of psychology, 10(2), 458-467. https://doi.org/10.1017/S1138741600006727

McMullin, J. A. \& Cairney, J. (2004). Self-esteem and the intersection of age, class, and gender. Journal of aging studies, 18(1), 75-90. https://doi.org/10.1016/j.jaging.2003.09.006

Montag, C., Wegmann, E., Sariyska, R., Demetrovics, Z., \& Brand, M. (2019). How to overcome taxonomical problems in the study of Internet use disorders and what to do with "smartphone addiction"?. Journal of behavioral addictions, 1-7. https://doi.org/10.1556/2006.8.2019.59

Nayak, J. K. (2018). Relationship among smartphone usage, addiction, academic performance and the moderating role of gender: A study of higher education students in India. Computers \& Education, 123, 164-173. https://doi.org/10.1016/j.compedu.2018.05.007

O'Dea, S. (2021). Number of smartphone users worldwide from 2016 to 2023. Statista. Recuperado de https://www.statista.com/statistics/330695/number-of-smartphone-users-worldwide/

ONTSI. (2019). La sociedad en red. Transformación digital en España. Informe Anual 2018. Recuperado de https://www.ontsi.red.es/sites/ontsi/files/2019-10/InformeAnualLaSociedadEnRedEdic2019.pdf

Oraison, H., Nash-Dolby, O., Wilson, B., \& Malhotra, R. (2020). Smartphone distraction-addiction: Examining the relationship between psychosocial variables and patterns of use. Australian Journal of Psychology, 72(2), 188-198. https://doi.org/10.1111/ajpy.12281

Panova, T. \& Carbonell, X. (2018). Is smartphone addiction really an addiction?. Journal of behavioral addictions, 7(2), 252-259. https://doi.org/10.1556/2006.7.2018.49

Peplau, L. A. \& Perlman, D. (1982). Perspectives on loneliness. In Peplau, L. A., Perlman, D. (Eds.), Loneliness: A sourcebook of current theory, research and therapy (pp. 1-8). New York: Wiley.

Peplau, L. A., Russell, D., \& Heim, M. (1979). The experience of loneliness. In I. Frieze, D. Bar-Tal \& J. S. Carroll (Eds.), New approaches to social problems: Applications of attribution theory (pp.53-78). San Francisco: Jossey-Bass.

Podsakoff, P. M., MacKenzie, S. B., Lee, J. Y., \& Podsakoff, N. P. (2003). Common method biases in behavioral research: a critical review of the literature and recommended remedies. Journal of applied psychology, 88(5), 879. https://doi.org/10.1037/0021-9010.88.5.879

Puskar, K. R., Marie Bernardo, L., Ren, D., Haley, T. M., Hetager Tark, K., Switala, J., \& Siemon, L. (2010). Self-esteem and optimism in rural youth: Gender differences. Contemporary Nurse, 34(2), 190-198. https://doi.org/10.5172/conu.2010.34.2.190

Randler, C., Wolfgang, L., Matt, K., Demirhan, E., Horzum, M. B., \& Beşoluk, Ş. (2016). Smartphone addiction proneness in relation to sleep and morningness-eveningness in German adolescents. Journal of behavioral addictions, 5(3), 465-473. https://doi.org/10.1556/2006.5.2016.056

Romero-Rodríguez, J. M., Aznar-Diaz, I., Marin-Marin, J. A., Soler-Costa, R., \& Rodríguez-Jiménez, C. (2020). Impact of problematic smartphone use and Instagram use intensity on self-esteem with university students from physical education. International journal of environmental research and public health, 17(12), 4336. https://doi.org/10.3390/ijerph17124336

Rosenberg, M. (1965). Society and adolescent self-image. New Jersey: Princeton University Press. https://doi.org/10.1515/9781400876136

Segrin, C. (2019). Indirect effects of social skills on health through stress and loneliness. Health communication, 34(1), 118-124. https://doi.org/10.1080/10410236.2017.1384434

Sha, P., Sariyska, R., Riedl, R., Lachmann, B., \& Montag, C. (2019). Linking internet communication and smartphone use disorder by taking a closer look at the Facebook and WhatsApp applications. Addictive behaviors reports, 9, 100148. https://doi.org/10.1016/j.abrep.2018.100148

Thomée, S. (2018). Mobile phone use and mental health. A review of the research that takes a psychological perspective on exposure. International journal of environmental research and public health, 15(12), 2692. https://doi.org/10.3390/ijerph15122692

Trzesniewski, K. H., Donnellan, M. B., \& Robins, R. W. (2013). Development of self-esteem. In V. Zeigler-Hill (Ed.), Self-esteem (pp. 60-79). New York: Psychology Press.

Van Deursen, A. J., Bolle, C. L., Hegner, S. M., \& Kommers, P. A. (2015). Modeling habitual and addictive smartphone behavior: The role of smartphone usage types, emotional intelligence, social stress, selfregulation, age, and gender. Computers in human behavior, 45, 411-420. https://doi.org/10.1016/j.chb.2014.12.039

Wang, H. Y., Sigerson, L., Jiang, H., \& Cheng, C. (2018). Psychometric properties and factor structures of Chinese smartphone addiction inventory: Test of two models. Frontiers in psychology, 9, 1411. https://doi.org/10.3389/fpsyg.2018.01411

Wang, P., Zhao, M., Wang, X., Xie, X., Wang, Y., \& Lei, L. (2017). Peer relationship and adolescent smartphone addiction: The mediating role of self-esteem and the moderating role of the need to belong. Journal of behavioral addictions, 6(4), 708-717. https://doi.org/10.1556/2006.6.2017.079

Weiser, E. B. (2000). Gender differences in Internet use patterns and Internet application preferences: A twosample comparison. Cyberpsychology and behavior, 3(2), 167-178. https://doi.org/10.1089/109493100316012

Wong, N. M., Yeung, P. P., \& Lee, T. M. (2018). A developmental social neuroscience model for understanding $\begin{array}{lllll}\text { loneliness in adolescence. Social neuroscience, } & 13(1), & 94-103 .\end{array}$ https://doi.org/10.1080/17470919.2016.1256832

World Health Organization. (2015). Public health implications ofexcessive use of the Internet, computers, smartphones and similar electronic devices. Recuperado de https://apps.who.int/iris/handle/10665/184264 
Hidalgo-Fuentes, S. / Psicodebate, 21(2), $50-60$.

World Health Organization. (2018). International Classification of Diseases 11th Revision (ICD-11). Recuperado de https://icd.who.int/

Yang, Z., Asbury, K., \& Griffiths, M. D. (2019). An exploration of problematic smartphone use among Chinese university students: Associations with academic anxiety, academic procrastination, self-regulation and subjective wellbeing. International Journal of Mental Health and Addiction, 17(3), 596-614. https://doi.org/10.1007/s11469-018-9961-1

Yárnoz, S. (2008). Adaptación al castellano de la escala para la evaluación de la soledad social y emocional en adultos SESLA-S. International Journal of Psychology and Psychological Therapy, 8(1), 103-116.

Recibido 06-04-2021 | Aceptado 11-05-2021 